LA-8421-MS

Informal Report

UC-38

Issued: Octoiser 1980

\title{
The BDD: A Dosimeter for the Global Positioning System
}

Harold V. Argo

Daniel N. Baker

Richard D. Belian

Leroy K. Cope

Paul R. Higbie

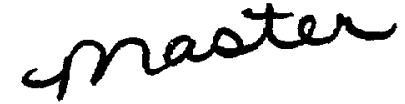




\title{
THE BDD: A DOSIMETER FOR THE GLOBAL POSITIONING SYSTEM
}

\author{
by \\ Harold V. Argo, Daniel N. Baker, Richard D.Belian, \\ Leroy K. Cope, and Paul R. Higbie
}

\begin{abstract}
This report describes the design and operation of the BDD, a four-channel spectrometer carried by some satellites of the Global Positioning System to collect data about magnetically trapped particle fluxes. The methods of data collection and analysis are also discussed.
\end{abstract}

\section{INTRODUCTION}

Satellites of the US Air Force Global Positioning System (GPS), which are in highly inclined (55-63 circular orbits at an altitude of 11000 nautical miles. pass twice per 12-h orbit near the peak-intensity region of magnetically trapped energetic particles. Radiation damage to the satellite components would be detrimental to the system's planned longevity. Available experimental or theoretical knowledge of particle fluxes at GPS orbits is inadequate for predicting the extent of radiation damage, largely because the resident trapped particle populations vary and, in addition, depend on the level of solar activity.

A secondary mission of the GPS program, beginning with the launch of the satellite FSV-6 in late 1980, is to carry instrumentation in support of the Integrated Operational Nuclear-Test Detection System program. This instrumentation includes an $\mathrm{x}$-ray burst detector (BDX) and a logic-control and data-storage unit (BDP). It was decided that the simplest and most effective way to obtain the required particle-flux data is to equip some satellites with an appropriate dosimeter rather than a BDX. Because this decision was made when the BDP and the $\mathrm{BDX}$ were already in the hardware stage, restrictions were placed on the dosimeter (BDD) design.
- The shape, weight, and power requirements of the BDD were to be the same as those of the BDX.

- The BDD was to be connected to the BDP with the same cable that connects the BDX to the BDP.

- The BDD data format was to be fitted into the existing BDX data train stored in the BDP.

These requirements have been met with no change in the BDP hardware and with the addition of only a single hard wire to the connecting cable. The level on this wire determines the subset of BDP firmware appropriate to processing data from a BDX or a BDD.

\section{DOSIMETER CONCEPT AND DESIGN}

For the purpose at hand, the most useful form of dosimeter data is energy spectra with absolute intensity as a function of time and position in orbit. From such data one can calculate the radiation dose received by a satelite component and so predict the expected degradation of its performance with time. Within the design restrictions mentioned above, we have devised a four-channel spectrometer that will provide electron-flux data over the energy range from 0.3 to $10 \mathrm{MeV}$ and proton-flux data from 6 to $100 \mathrm{MeV}$. 
Figure 1 shows simplified sketches of the BDD package and detector geometry. A $700-\mu$-thick surface-barricr silicon sensor in each of the four channels is protected from the full radiation intensity by a thick hemispherical dome. Particles are admitted to the sensor through cylindrical, radially oriented collimating apertures in the dome. Thin hemispherical filters covering the bottoms of the collimating apertures determine the energy range covered by each channel. This "saltshaker" design permits the detectors to sample uniformly a large fraction of one hemisphere (and thus to measure the average omnidirectional flux) and at the same time shields the sensors from more than $99.2 \%$ of the incident particle flux.

The protective dome consists of an outer shell of aluminum (replaced at very low angles to the satellite surface by (ungsten) and an inner shell of gold. The thickness of aluminum was chosen to stop electrons while minimizing bremsstrahlung from them. The gold was chosen to stop very energetic electrons since not enough space was availabie to use aluminum exclusively. The tungsten section provides a well-defined polar angle for calculating the response of the detector to particles that leak through the aluminum/gold shielding.
The diameters of the collimating apertures in each dome have been matched to the threshold energy of that channel so that the expected dose rates in all channels will be comparable.

The thin hemispherical filters, which determine the energy range of each channel, are of aluminum. iron. or tungsten. Equivalent aluminum thicknesses of 10, 25.60 (2! mils of iron). and 135 (20 mils of tungsten) mils provide electron energy thresholds (measured) of 0.31 . 0.57 . 1.05, and $1.45 \mathrm{MeV}$. respectively, and proton energy thresholds of 6, 10, 17, and $27 \mathrm{MeV}$, respectively.

The four energy channels have been fitted onto the existing surface plate of the BDX unit; the associated power supply. amplifiers, discriminators, and data-processing logic have been fitted into the existing BDX container. Care was taken to position the collimating apertures so that they are not aligned with any satellite antennas or other fixtures occupying the same satellite face.

Table I lists various design parameters for the four channels of the BDD. The photograph of Fig. 2 shows the four detectors of the BDD; that of Fig. 3 shows the electronics package.
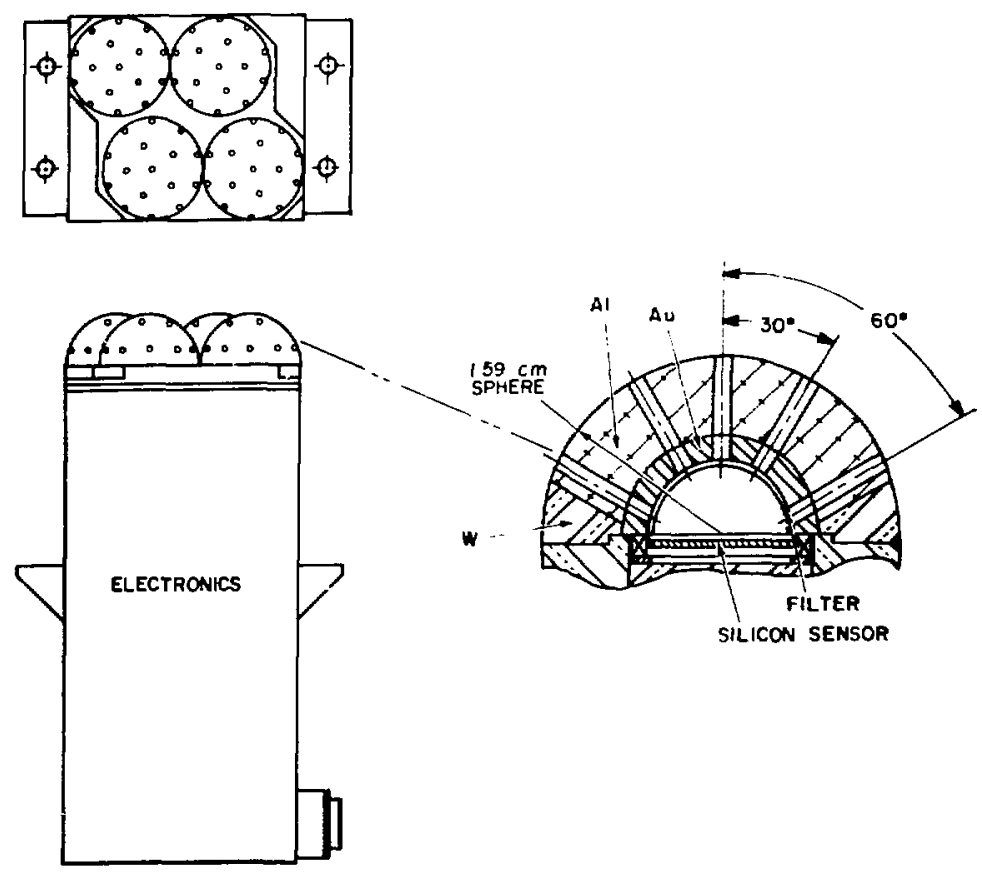

Fig. 1.

Simplified sketches of the BDD package and detector geometry. 


\section{TABLE I}

\section{DESIGN PARAMETERS OF THE}

FOUR BDD ENERGY CHANNELS

\begin{tabular}{ccccccc}
$\begin{array}{c}\text { Filter } \\
\text { Thickness } \\
\text { (mils) }\end{array}$ & $\begin{array}{c}\text { Electron } \\
\text { Threshold } \\
(\mathrm{MeV})\end{array}$ & $\begin{array}{c}\text { Proton } \\
\text { Threshold } \\
(\mathrm{MeV})\end{array}$ & $\begin{array}{c}\text { Effective } \\
\text { Solid Angle } \\
\left(\mathrm{cm}^{2} \cdot \mathbf{s r}\right)\end{array}$ & $\begin{array}{c}\text { Estimated } \\
\text { Electron } \\
\text { Dose Rate }^{\mathrm{b}} \\
(\mathrm{MeV} / \mathrm{s})\end{array}$ & $\begin{array}{c}\text { Maximum } \\
\text { Proton Rate }^{\mathrm{c}} \\
\left(\mathrm{s}^{-1}\right)\end{array}$ & $\begin{array}{c}\text { Estimated } \\
\text { Proton Dose Rate } \\
(\mathrm{MeV} / \mathrm{s})\end{array}$ \\
\hline & & & & & & \\
10 & 0.31 & 6 & 0.0029 & 29000 & 510 & 32000 \\
25 & 0.57 & 10 & 0.00434 & 26000 & 380 & \\
60 & 1.05 & 17 & 0.00976 & 28000 & 440 & \\
135 & 1.45 & 27 & 0.0392 & 32000 & 1190 & \\
\hline
\end{tabular}

${ }^{a}$ Equivalent thickness of aluminum.

bAs predicted by the model used by Roeske.

'For the 4-9 August 1972 soiar proton event.

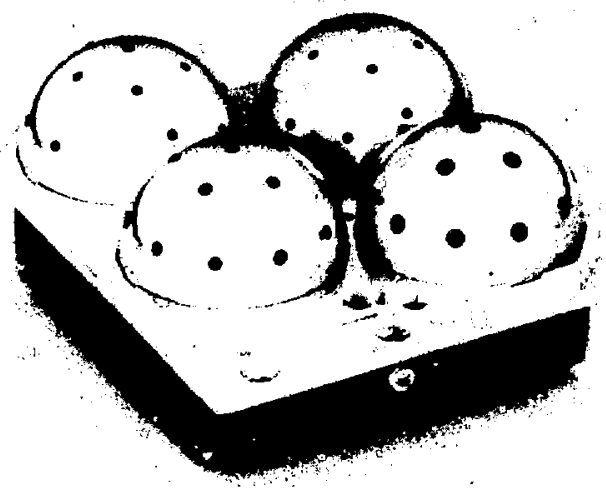

Fig. 2.

Photograph of the four BDD detectors.

Figure 4 shows two idealized sets of detector energy-response curves of the four channels, one for electrons and one for protons. The energy threshold of each channel is determined by the entrance-filter thickness. As the energy of the incident particle increases, the energy deposited in the sensor increases monotonically until the energy threshold for penetration of the sensor is reached. A sharp break in the energy deposition curve occurs at that energy, followed by a decrease as the energy of the particle approaches the minimum ionizing level. The maximum energy that an electron can deposit in the sensor is slightly greater than $0.4 \mathrm{MeV}$; the

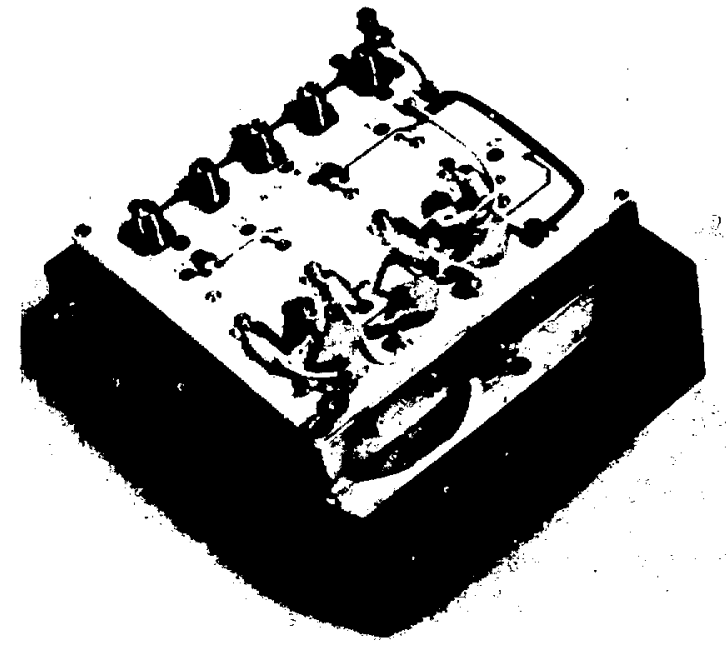

Fig. 3.

Photograph of the BDD electronies package.

maximum energy deposited by a proton is approximately $10 \mathrm{MeV}$. An energy-level discriminator set near 0.5-0.6 $\mathrm{MeV}$ will effect separation of sensor pulses generated by electrons from those generated by protons with energies greater than $6 \mathrm{MeV}$. Since the electron fluxes are normally several orders of magnitude greater than the proton fluxes, there will be little contamination of the electron-nux data by protons with energies less than $\mathbf{0 . 5}$ $\mathrm{MeV}$ and no contamination of the proton-flux data by eiectrons. 


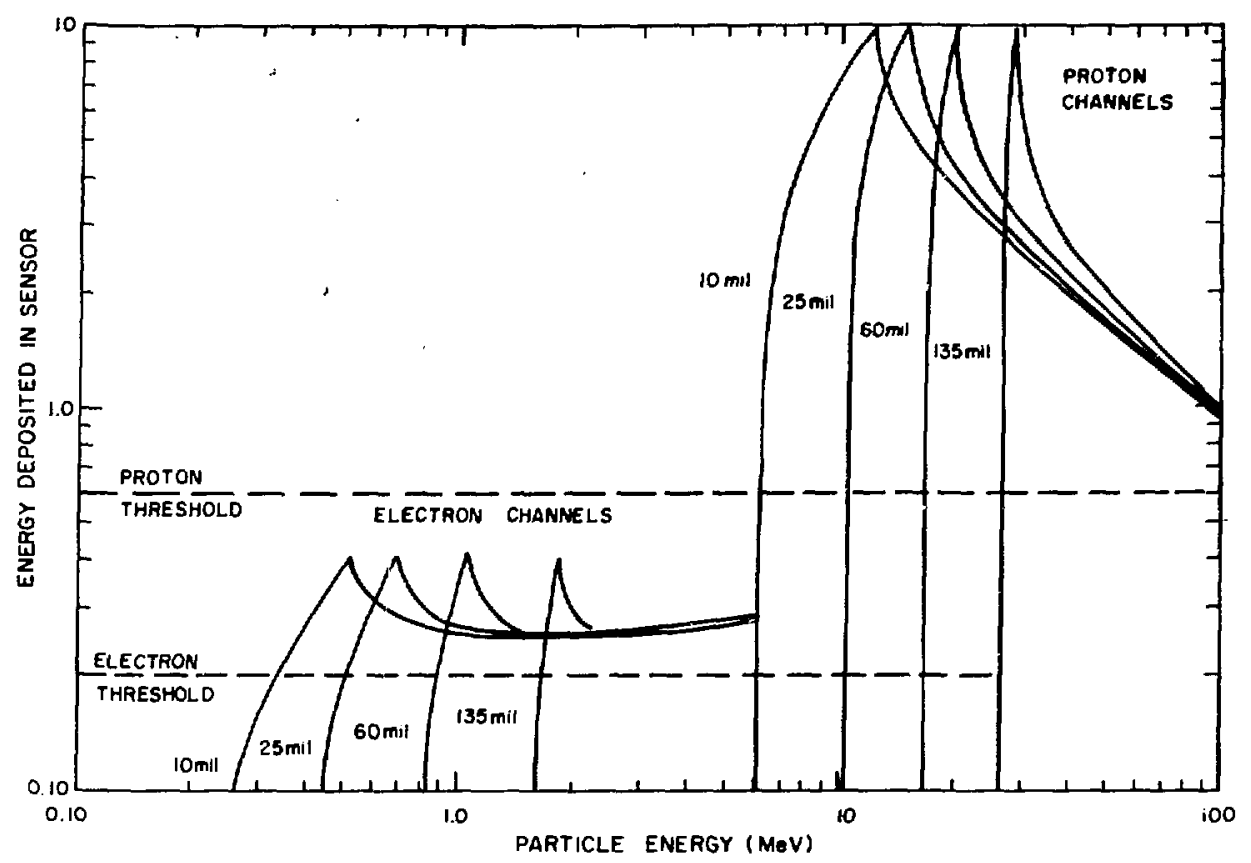

Fig. 4.

Idealized unit response functions for the four BDD channels. The thresholds are determined by the entrance fiker thicknesses and the peaks by the sensor thickness.

Figure 5 shows examples of the electron and proton energy spectra that we expect to be encountered by the BDD. The particle fluxes are highly dependent on solar activity and will vary rapidly with position in orbit. The clectron energy spectra are for equatorial orbits at an altitude of 11000 nautical miles and are given by the AE-4 model of Singley and Vette' or by a calculation of Roeske* who used a preliminary version of the AE-7 model. The AE-4 10\%-probability curve represents the electron energy spectrum that, according to this model, will be exceeded $10 \%$ of the time. The proton energy spectrum is that observed ${ }^{2}$ during the giant solar proton events of 4-9 August 1972, which are the most intense high-energy proton fluxes ever recorded.

\section{DATA ANALYSIS}

Each channel will contain the integrated energy deposited by all electrons (protons) with energies greater

\footnotetext{
* These data were furnished by S. B. Roeske of Sundia Laboratories, Albuquerque.
}

than its electron (proton) threshold energy. The usual technique for converting observed count rates into fluxes ${ }^{3}$ is to choose an effective energy threshold and a constant for the detector that minimize the effect of different energy spectra. Baker ${ }^{4}$ and Van Allen et al. ${ }^{5}$ gave a detailed exposition of this technique and coined for it the name "bow-tie analysis." In the case of regular spectra of the type encountered in magnctically trapped particle populations (Fig. 5), the technique can be applied to data taken over integrated energy intervals and can sccurately reproduce the original spectrum.

The basis for this bow-tie analysis is the assumption that we can replace the relatively complex response function of a detector with a simple effective response function. As illustrated in Fig. 6a, we will replace the true response $R(E)$ of a detector (solid curve) with an effective step-function response $R^{*}$ (dashed rectangular curve) characterized by the parameters $\Delta E^{*}$ (effective constant response) and $E_{T}$ (effective threshold energy). That is, we will set

$R(E)=0$ for $E<E_{T}$ 


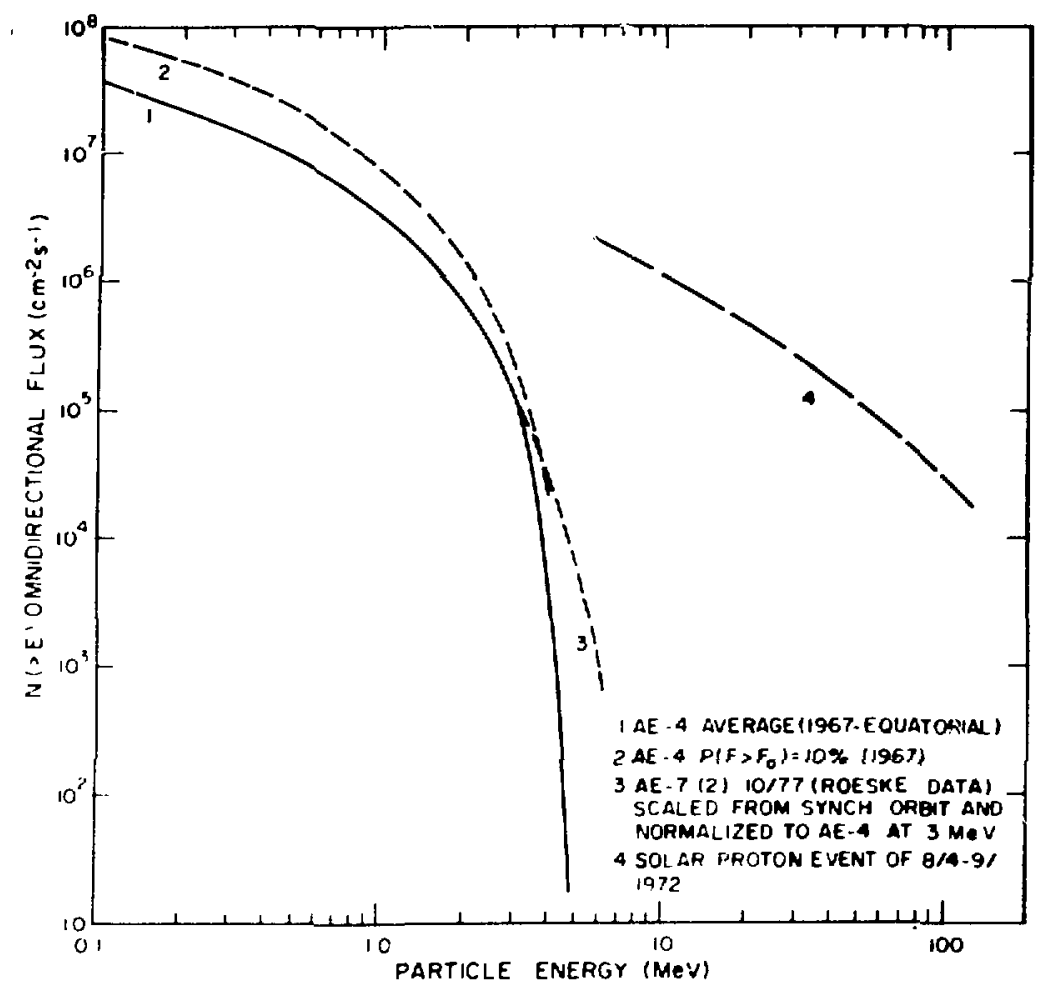

Fig. 5.

Energy spectra of trapped electrons at an altitude of 11000 nautical miles as predicted by current models and of the giant solar proton event of 4.9 August 1972.

and

$R(E)=\Delta E^{*}$ for $E \geq E_{T}$.

For each detector we will find an "optinum" parameter pair $\left(\Delta E^{*}, E_{T}\right)$ that will replicate its response over a wide range of incident-particle spectra.

To determine the optimum $\left(\Delta E^{*}, E_{T}\right)$, we establish the crespondence

$\Delta E^{*} \int_{E_{T}}^{\infty}(d J / d E) d E+\int_{0}^{\infty} R(E)(d J / d E) d E$

The expression on the right is the exact count rate expected for an energy spectrum given by $d J / d E$; it is determined by direct numerical integration of the product of the measured detector response $R(E)$ and the energy spectrum $\mathrm{dJ} / \mathrm{dE}$. The expression on the left is the integral of the product of the step-function response and the energy spectrum. The electron spectra that we expect to be encountered by the BDD (Fig. 5) are well represented by an exponential form such as

$\mathrm{dJ} / \mathrm{dE} \propto \exp \left(-E / E_{0}\right)$,

where $0.2 \mathrm{MeV} \leq \mathrm{E}_{0} \leq 1.0 \mathrm{MeV}$. Combining Eqs. 1 and 2 and expressing $\Delta E^{*}$ as a funciion of $E_{T}$, we obtain

$\Delta E^{*}=\frac{\int_{0}^{\infty} R(E) \exp \left(-E_{0} / E_{0}\right) d E}{\int_{E_{T}}^{\infty} \exp \left(-E / E_{0}\right) d E}$.

Using Eq. 3. we then plot $\Delta E^{*}$ vs $E_{\mathrm{T}}$ for various values of the spectral index $E_{0}$; we obtain a family of curves that 

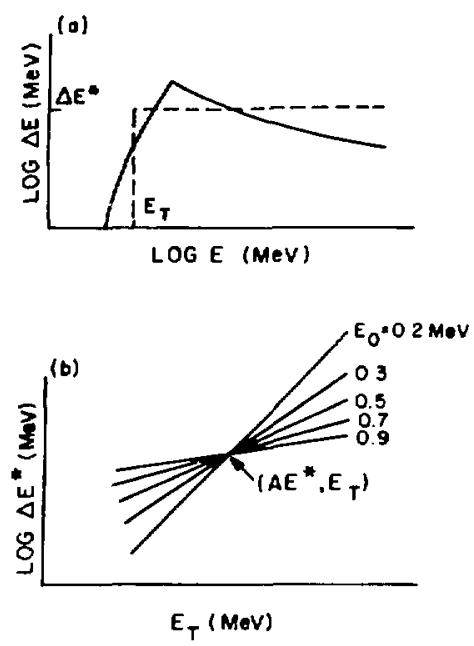

Fig. 6.

(a) Typical detector response function for an electron channet and the effective step-function response that is substituted for it in the bow-tie analysis.

(b) Family of curves cbtained by plotting $\Delta E^{*}$ vs $E_{\mathrm{T}}$ for various values of the spectral index $E_{0}$. The coordinates $\left(\Delta E^{*}, E_{T}\right)$ of the point of intersection characterize an effective step-function response of the detector that accurately reproduces the original energy spectrum over a wide range of spectral indices.

roughly intersect at a point to form a bow tie, as illustrated in Fig. 6b. The average intersection point is assumed to be the optimum parameter pair $\left(\Delta \mathrm{E}^{*}, \mathrm{E}_{\mathrm{T}}\right)$, which characterizes the effective response of the detector over a wide range of spectral indices. In the data analysis. the number of electrons in the spectrum above the threshold $E_{T}$ is found by dividing the total energy deposited in the detector by the effective energy deposited per particle $\Delta \mathrm{E}^{*}$.

Figure 7 summarizes the bow-tie analysis results for the four electron channels of the BDD.

\section{DATA COLLECTION}

Electrons and protons entering a detector through one of the collimating apertures will generate voltage pulses whose heights are proportional to the energy deposited in the sensor. The pulse height of each pulse (in MeV) is cumulatively stored in an appropriate eight-bit temporary-memory storage register of the BDD. There are eight such storage registers: four each for electron and
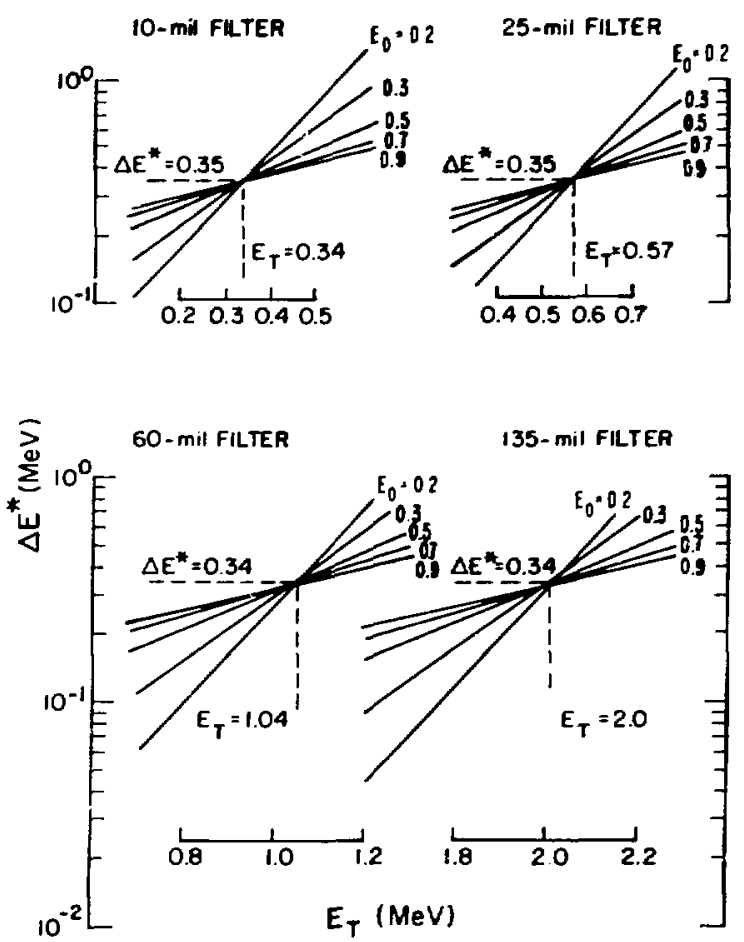

Fig. 7.

Results of bow-tie analyses for the four eleciron channets of the BDD.

proton pulse-heiglıt data. The pulses generated by electrons or protons are identified by pulse heights below or above. respectively, a predetermined level $(\sim 0.6 \mathrm{MeV})$.

Every $3.2 \mathrm{~min}$ the accumulated data in either the electron or proton storage registers is transferred and stored sequentially in the BDD portion of the BDP event memory. Every fourth time interval the BDD frame sync and satellite $\mathrm{Z}$ time are stored with the data. This block of data. four collection intervals plus $Z$ time and frame sync. makes up a BDD data frame (BDDF) in event memory. A BDDF contains 24 eight-bit words; its detailed format is shown in Fig. 8.

The $\mathbf{Z}$ time is that of the immediately following BDDI. The BDD5X ( $\mathrm{X}=\mathrm{A}, \mathrm{B}, \mathrm{C}, \mathrm{D})$ are status words that indicate whether the data were generated by elecsrons or protons and specify the data collection mode and the upper and lower proton-rate thresholds.

There are three basic modes of data colkection, selected by commutated XCALI command.

- 3/0 The electron channels are read out every 3.2 min. 


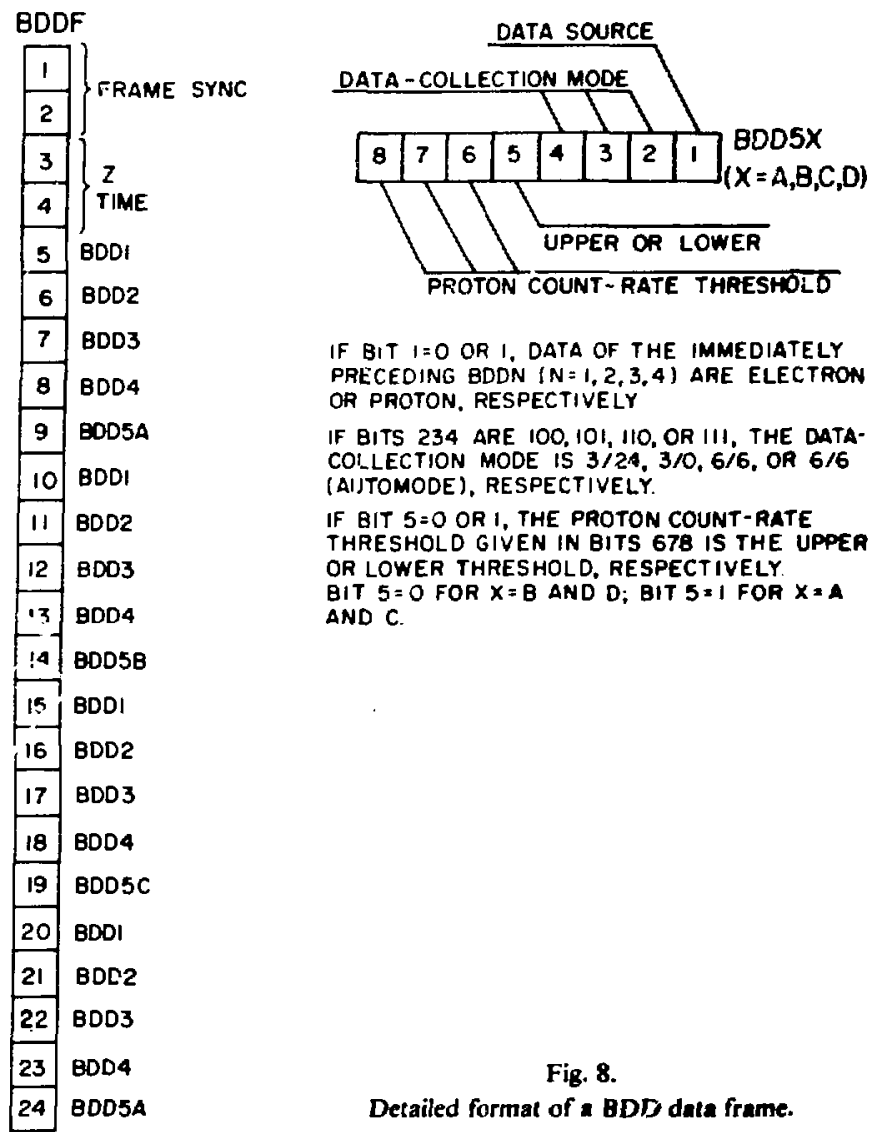

- 6/6 The electron and proton channels are read out alternately every $3.2 \mathrm{~min}$.

- 3/24 The electron channels are read out every 3.2 min for seven intervals; then the accumulated proton channels are read out at the end of the eighth interval.

Since there will be long periods during which the proton channels will be counting at or near background levels. the $3 / 0$ and $3 / 24$ modes will be used extensively. If a solar proton event should occur while data is being collected in either of these modes. circuitry is provided (see Sec. V) that will automatically change the data-collection mode. An upper proton-rate threshold, selected by commutated XCALI command from eight possible levels, effects a shift of the mode from $3 / 0$ or $3 / 24$ to $6 / 6$ (automode) when the proton rate exceeds this threshold. A lower proton-rate threshold, again selected by commutated XCALI command from eight possible levels, effects return to the original mode when the proton rate falls below this threshold. Table II lists both the count rate and the accumulated count per 3.2 min interval associated with each of the eight upper and lower proton-rate thresholds.

The 4096 words of BDP event memory allocated for storage of BDDF will accommodate $36 \mathrm{~h}$ of data collection. It is planned to transmit the BDD data to ground on L3 every $24 \mathrm{~h}$ through an event memory readout.

\section{CIRCUIT DESIGN}

Figure 9 is a block diagram of the BDD circuits. The system consists of four surface-barrier silicon detectors with associated channels of anakg electronics and data-handling logic. A detector pulse is amplified by a charge-sensitive preamplifier, which feeds in parallel an electron-level detector (ELD), a proton-kvel detector (PLD), and a pulse height-to-time converter that gates $4-\mathrm{MHz}$ pulses, the number of which is proportional to the incident-particle energy. 
TABLE II

\section{PROTON COUNT-RATE THRESHOLDS}

\begin{tabular}{|c|c|c|c|c|}
\hline \multirow[b]{2}{*}{$\begin{array}{l}\text { Threshold } \\
\text { Designation }\end{array}$} & \multicolumn{2}{|c|}{$\begin{array}{c}\text { Upper } \\
\text { Threshold }\end{array}$} & \multicolumn{2}{|c|}{$\begin{array}{l}\text { Lower } \\
\text { Threshold }\end{array}$} \\
\hline & $\begin{array}{l}\text { Accumulated Count } \\
\text { per } 3.2 \mathrm{~min}\end{array}$ & $\begin{array}{c}\text { Count Rate } \\
\left(\mathrm{s}^{-1}\right)\end{array}$ & $\begin{array}{l}\text { Accumulated Count } \\
\text { per } 3.2 \mathrm{~min}\end{array}$ & $\begin{array}{c}\text { Count Rate } \\
\left(5^{-1}\right)\end{array}$ \\
\hline 1 & 16400 & 85 & 512 & 2.67 \\
\hline 2 & 32800 & 171 & 1024 & 5.34 \\
\hline 3 & 65600 & 342 & 2048 & 10.7 \\
\hline 4 & 128000 & 684 & 4096 & 21.4 \\
\hline 5 & 256000 & 1370 & 8192 & 42.7 \\
\hline 6 & 512000 & 2730 & 16400 & 85.4 \\
\hline 7 & 1024000 & 5470 & 32800 & 171 \\
\hline 8 & 2048000 & 10940 & 65600 & 342 \\
\hline
\end{tabular}

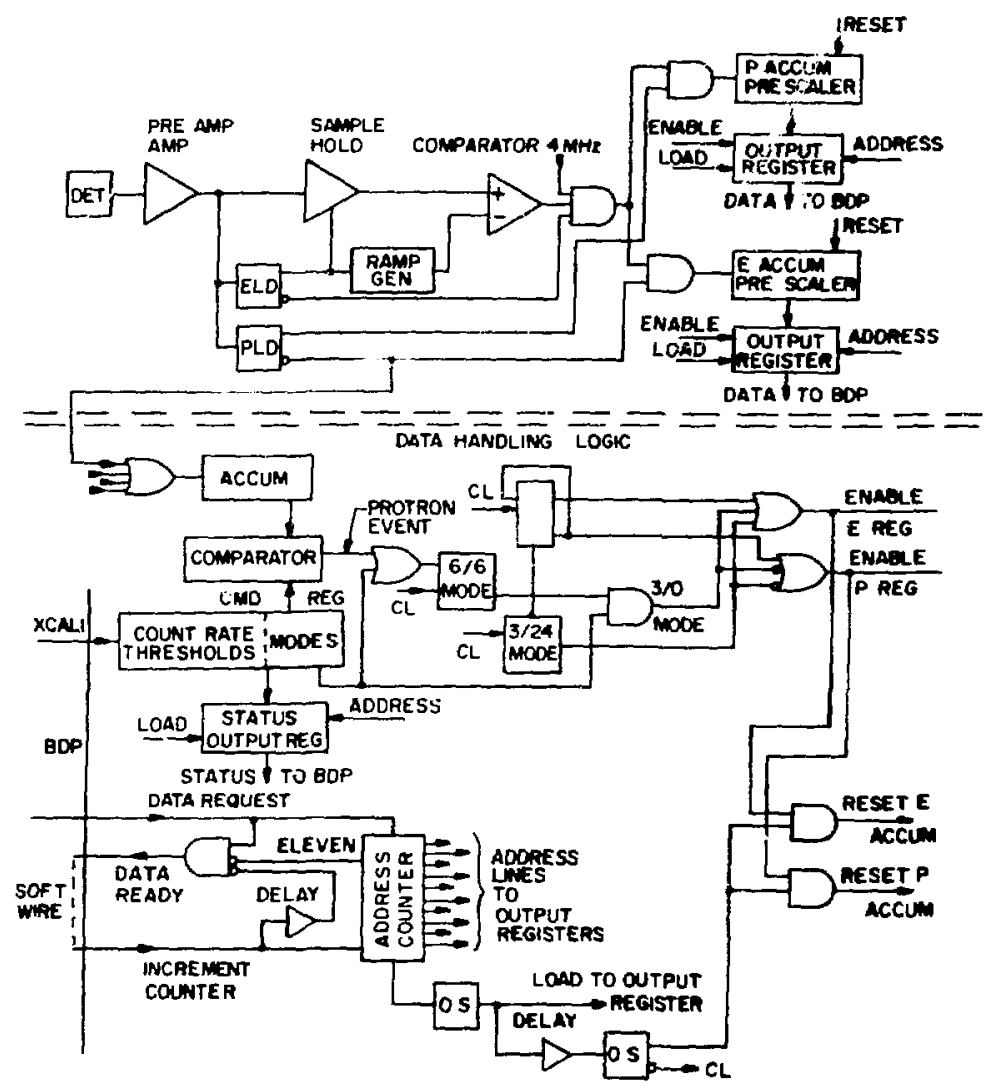

Fit.9.

Block diagram of the BDD electronic circuits. 
Triggering of the ELD upon receipt of a pulse inhibits the comparator gate and closes an analog switch to a sample-hold amplifier whose output is the positive input of the comparator. The negative input of the comparator is supplied by a ramp generator. A closed analog switch in the feedback path of the ramp generator results in a low gain (small positive voltage). The output of the comparator gate goes positive when the ELD pulse terminates. and the high comparator output initiates the gating of $4-\mathrm{MHz}$ pulses. Absence of the ELD pulse opens the feedback path of the ramp generator and thus causes the gain to increase. The output of the ramp generator goes positive and is slowed by the charging of the feedback capacitor. At the point where the output of the ramp generator has gone as positive as the analog signal from the sample-hold amplifier, the output of the comparator goes to zero, and the $4-\mathrm{MHz}$ gate is inhibited. The number of gated $4-\mathrm{MHz}$ pulses is thus propurtional to pulse height. If the amplifier pulse is large enough to trigger the PLD also, the electron $4-\mathrm{MHz}$ gate is inhibited and the proton $4-\mathrm{MHz}$ gate is enabled: the pulses are thus routed to the proton accumulator.

The output of the analog circuitry is a number of gated 4- $\mathrm{MHz}$ pulses on two lines, electron and proton, with the number of pulses proportional to the energy deposited in the detector. The digital circuitry accumulates these pulses, stores them until the BDP requests data (every $3.2 \mathrm{~min}$ ), and then transfers them to BDP memory. The accumulated pulses are prescaled (bit-compressed-15 bits to 8 bits).

When data are requested by the BDP, the output register is read out. and the data currently being accumulated is shifted into temporary store in the output register. The accumulator is reset, and accumulation is started for the next time interval.

Data from all four proton channels are accumulated by a separate scaler and compared with a preselected upper count-rate threshold. If the proton count rate exceeds the selected level. the current data-collection mode is changed to 6/6 (automode). Dats continue to be collected in this modc until the proton count rate falls below a preselected lower count-rate threshold; at this point. the data-collection mode is returned to the original mode. An eight-bit command register is incorporated in the system to enable selection of the data-collection mode and of the upper and lower proton count-rate thresholds.

\section{REFERENCES}

1. G. W. Singley and J. I. Vette, "The AE-4 Model of the Outer Radiation Zone Electron Environment," National Space Science Data Center report NSSDC 72-06 (August 1972).

2. J. H. King. "Solar Proton Fluences for 1977-1983 Space Missions," J. Spacecr. Rockets 11, 401 (1974).

3. W. L. Kraushaar, G. W. Clark, G. P. Garmire, R. Borken. P. Higbie. C. Leong, and T. Thorsos, "High-Energy Cosmic Gamma-Ray Observations from the OSO-3 Satellite." Astrophys. J. 177, 341 (1972).

4. D. N. Baker, "Energetic Particle Fluxes and Spectra in the Jovian Magnetosphere," Ph.D. thesis, University of lowa. 1974.

5. J. A. Van Allen. D. N. Baker, B. A. Randall, and D. D. Sentman. "The Magnetosphere of Jupiter as Observed with Pioneer 10. 1. Instrument and Principal Findings," J. Geophys. Res. 79, 3559 (1974). 